\title{
Detection of Antimicrobial Resistance Genes Associated with Carbapenem Resistance from the Whole-Genome Sequence of Acinetobacter baumannii Isolates from Malaysia
}

\author{
Mohan Rao ${ }^{\mathbb{D}},{ }^{1}$ Fairuz A. Rashid, ${ }^{1}$ Surianti Shukor, ${ }^{1}$ Rohaidah Hashim $\mathbb{D},{ }^{1}$ \\ and Norazah Ahmad ${ }^{2}{ }^{2}$ \\ ${ }^{1}$ Bacteriology Unit, Infectious Disease Research Centre, Institute for Medical Research, Ministry of Health, \\ Kuala Lumpur, Malaysia \\ ${ }^{2}$ Infectious Disease Research Centre, National Institute of Health, Ministry of Health, Shah Alam, Malaysia \\ Correspondence should be addressed to Mohan Rao; mogaeviridae@gmail.com
}

Received 7 October 2019; Revised 20 December 2019; Accepted 9 January 2020; Published 2 April 2020

Academic Editor: José Ramón Blanco

Copyright (c) 2020 Mohan Rao et al. This is an open access article distributed under the Creative Commons Attribution License, which permits unrestricted use, distribution, and reproduction in any medium, provided the original work is properly cited.

Background. The spread of carbapenem-resistant A. baumannii $(\mathrm{CrAb})$ is gaining worldwide attention. The spread of this pathogen is largely due to its ability to acquire various resistance genes of intrinsic and extrinsic origins that confer unpredictable susceptibility to $\beta$-lactams. The aim of this study was to analyze $\beta$-lactamase genetic compositions of CrAb in Malaysia. Methods. Whole-genome sequencing (WGS) was carried out on $13 \mathrm{CrAb}$ isolates from clinical samples in Malaysia from 2011 to 2016. Results. Endotracheal aspirate was the dominant clinical sample source $(n=6)$, and only one isolate was obtained from wound swab. A total of 6 sequence types (STs) of the Oxford scheme were identified, including 4 reported STs and 2 novel STs. Eleven isolates were classified into clonal complex 92 (CC92/ICII), among which ST195 and ST208 were the most prevalent STs. All 13 CrAb isolates harbored multiple $\beta$-lactamase genes. bla $a_{\text {OXA-23 }}(n=13)$ and $b a_{\text {OXA-66 }}(n=11)$ were the dominant carbapenemase gene families found in these isolates. All isolates harbor $b l a_{\mathrm{ADC}}, b l a_{\mathrm{OXA}-51-l i k e}$, and $b l a_{\mathrm{OXA}-23-\text { like }}$ genes. $b l a_{\mathrm{TEM}}(n=7), b l a_{\mathrm{NDM}-1}(n=3), b l a_{\mathrm{CARB}-8}(n=1)$, and $b l a_{\mathrm{PER}-3}$ $(n=1)$ are amongst other $\beta$-lactamase genes found in this study. ISAba1 was found upstream to $b l a_{\text {OXA-23 }}(n=13), b l a_{\text {OXA-66 }}(n=1)$, and $b l a_{\mathrm{ADC}}(n=11)$. All bla $a_{\mathrm{NDM}-1}$ isolates had ISAba125 (mobile genetic element) upstream to the genes. All isolates were positive for Tn2006/2008 and Tn2009 but were negative for Tn2007. Conclusion. Most of the isolates were grouped under the CC92 clonal complex which belongs to international clonal lineage 2 . These findings predict that carriage of carbapenem-resistant genes possibly constitutes the underlying basis of high level of international clone II prevalence. Therefore, molecular surveillance and antimicrobial stewardship are essential in implementing policies to prevent and control the spread of CrAb in hospital settings.

\section{Introduction}

Acinetobacter baumannii (A. baumannii) is an infectious agent that has been the leading cause of hospital-acquired infections [1]. It is an opportunistic pathogen that poses significant threat to public health and associated with high mortality [2]. A. baumannii nosocomial infection is now common throughout the world [3, 4]. Selection of an appropriate empirical antimicrobial agent is extremely difficult due to its unpredictable antimicrobial resistance genes which are commonly acquired via mobile genetic elements [5].
A. baumannii belongs to a group of clinically important organism, known as ESKAPE. It is predominantly found among health care-associated organisms that have the potential of substantial antibiotic resistance [6]. A. baumannii infection usually involves excretory organ systems that contain high level of fluids. The most common sites of infection are respiratory tract, urinary tract, and peritoneal cavity and highly associated with indwelling devices such as endotracheal tube, urinary catheter, Tenckhoff catheter, and intravascular catheter [7].

Carbapenem-resistant A. baumannii (CrAb) was identified as the critical organism based on the global priority 
TABLE 1: Study isolates, specimen types, biosample no., and GenBank accession number for A. baumannii isolates.

\begin{tabular}{lccc}
\hline Isolate & Specimen type & NCBI biosample no. & GenBank accession no. \\
\hline A. baumannii CRE1071/16 & Pus & SAMN11513371 & SWLT00000000 \\
A. baumannii CRE1159/16 & Urine & SAMN11513372 & SWLS00000000 \\
A. baumannii CRE157/16 & Urine & SAMN11513373 & SWLR00000000 \\
A. baumannii CRE158/16 & Endotracheal aspirate & SAMN11513374 & SWLQ00000000 \\
A. baumannii CRE245/15 & Rectal swab & SAMN11513375 & SWLP00000000 \\
A. baumannii CRE341/15 & Urine & SAMN11513376 & SWLO00000000 \\
A. baumannii CRE400/16 & Endotracheal aspirate & SAMN11513377 & SWLN00000000 \\
A. baumannii CRE449/14 & Endotracheal aspirate & SAMN11513378 & SWLM00000000 \\
A. baumannii CRE596/14 & Endotracheal aspirate & SAMN11513379 & SWLL00000000 \\
A. baumannii CRE645/15 & Rectal swab & SAMN11513380 & SWLK00000000 \\
A. baumannii CRE648/15 & Endotracheal aspirate & SAMN11513381 & SWLJ000000000 \\
A. baumannii CRE85/16 & Urine & SAMN11513382 & SWLI000000000 \\
A. baumannii CRE98/14 & Endotracheal aspirate & SAMN11513383 & SWLH00000000 \\
\hline
\end{tabular}

pathogen list proposed by the World Health Organization (WHO). It has been concluded that development of new antimicrobial is the current focus globally [8]. CrAb has become a major concern among healthcare facilities due to its rising prevalence. In countries of the Arab League and Vietnam, prevalence of $\mathrm{CrAb}$ has been reported ranging from 50 to $88 \%$, whereas in the United Kingdom, it ranges from 40 to $70 \%[9,10]$. According to the National Surveillance Antibiotic Resistance database, $\mathrm{CrAb}$ prevalence in Malaysia ranges from 50 to $60 \%$ and remained static since year 2008 up to 2016 [11]. However, several studies from different hospitals in Malaysia showed CrAb prevalence higher than the national surveillance [12].

Nonjudicious use of antibiotics has led A. baumannii to rapidly acquire antimicrobial resistance genes from the environment. At the same time, selective antimicrobial pressure induces genome rearrangement associated with chromosomally (intrinsic) encoded antimicrobial resistance genes which has resulted in transposition of insertion sequence (IS) as a promoter of various CHDLs [13]. A. baumannii possesses $b l a_{\mathrm{OXA}-51-l i k e,}$ an intrinsic carbapenemhydrolysing oxacillinase gene. The expression of this gene may vary with the presence of ISAbal as a promoter [14]. It also acquires certain $b l a_{\text {OXA }}$ and $b l a_{\text {non-OxA group genes }}$ from plasmids [15]. Predominantly acquired bla $a_{\text {OXA-group }}$ gene is $b l a_{\text {OXA-23-like, whereas the most prevalent } b l a_{\text {non-OXA }}}$ group gene is bla $a_{\mathrm{NDM}-1}[16]$.

This study is aimed to analyze the molecular characteristics of 13 A. baumannii isolates obtained from hospitalized patients in Malaysia with underlying carbapenemresistant phenotype.

\section{Methods}

Of 1933 A. baumannii isolates collected from various hospitals throughout Malaysia from year 2011 to 2016, we selected 13 carbapenem-resistant A. baumannii (CrAb) isolates that were resistant to carbapenems. No genes $\left(b l a_{\mathrm{NDM}}, b l a_{\mathrm{OXA}}, b l a_{\mathrm{KPC}}, b l a_{\mathrm{VIM}}\right.$, and $\left.b l a_{\mathrm{IMP}}\right)$ were found using in-house PCR. These isolates were recovered from patients receiving intensive care and were isolated from respiratory secretion, urine, rectal swabs, and pus. The initial identification test based on biochemical methods was performed using API 20E (bioMérieux, LaPlane, France). Antimicrobial susceptibility was determined by the disc diffusion method for gentamicin, amikacin, ciprofloxacin, cefepime, ceftazidime, aztreonam, imipenem, meropenem, ertapenem, and ampicillin-sulbactam according to Clinical and Laboratory Standards Institute (CLSI) criteria. Minimum inhibitory concentrations (MICs) of imipenem, meropenem, and ertapenem were determined by the E-Test method according to CLSI criteria. CrAb is defined as an $A$. baumannii isolate that is resistant to meropenem, ertapenem, and imipenem with an MIC value of $\geq 4 \mu \mathrm{g} / \mathrm{ml}$ via ETest. Table 1 summarizes study isolates, types of specimen, and the genebank identification.

Total DNA of these strains was extracted by using a MasterPure $^{\mathrm{TM}}$ DNA Purification Kit (Epicentre, Madison, Wisconsin, USA) and quantified using Qubit 2.0 ${ }^{\circledR}$ Fluorometer (Life Technologies, Carlsbad, CA). DNA libraries were prepared using a Nextera DNA Flex Library Prep Kit (Illumina Inc.), according to the manufacturer's instructions. Sequence data for all strains were obtained using an Illumina Nextseq platform (Illumina Inc., San Diego, CA, USA). Raw sequence quality trimming was carried out as described by SPAdes version 3.9.1 for de novo assembly and confirmation [17].

Average nucleotide identity (ANI) was calculated by using a gANI tool calculator, ANI calculator software version 1.0. ANI values above $95 \%$ between genomes of these isolates denote the same species [18]. Multilocus sequence typing (MLST) analysis was streamlined via the MLST program against PubMLST database via MLST version 2.6 software. Oxford scheme of A. baumannii was used for MLST analysis. The 7 housekeeping genes were $\operatorname{glt} A, \operatorname{gyr} B$, $g d h B, r e c A, c p n 60, g p i$, and $r p o D$ [19]. New alleles and STs were submitted to the curator of the database, and new ST numbers were allotted. Clonal complexes were assigned by eBURST and were defined as single locus and double-locus variants with an outgroup A. baumannii strain ATCC 17978 as a reference (GenBank accession number: CP000521.1) [20]. Antimicrobial resistance genes (AMR) were confirmed by the CARD and resistance gene identifier (Resfinder) via Abricate-Version 0.8 software [21]. kSNP version 3.0 was 


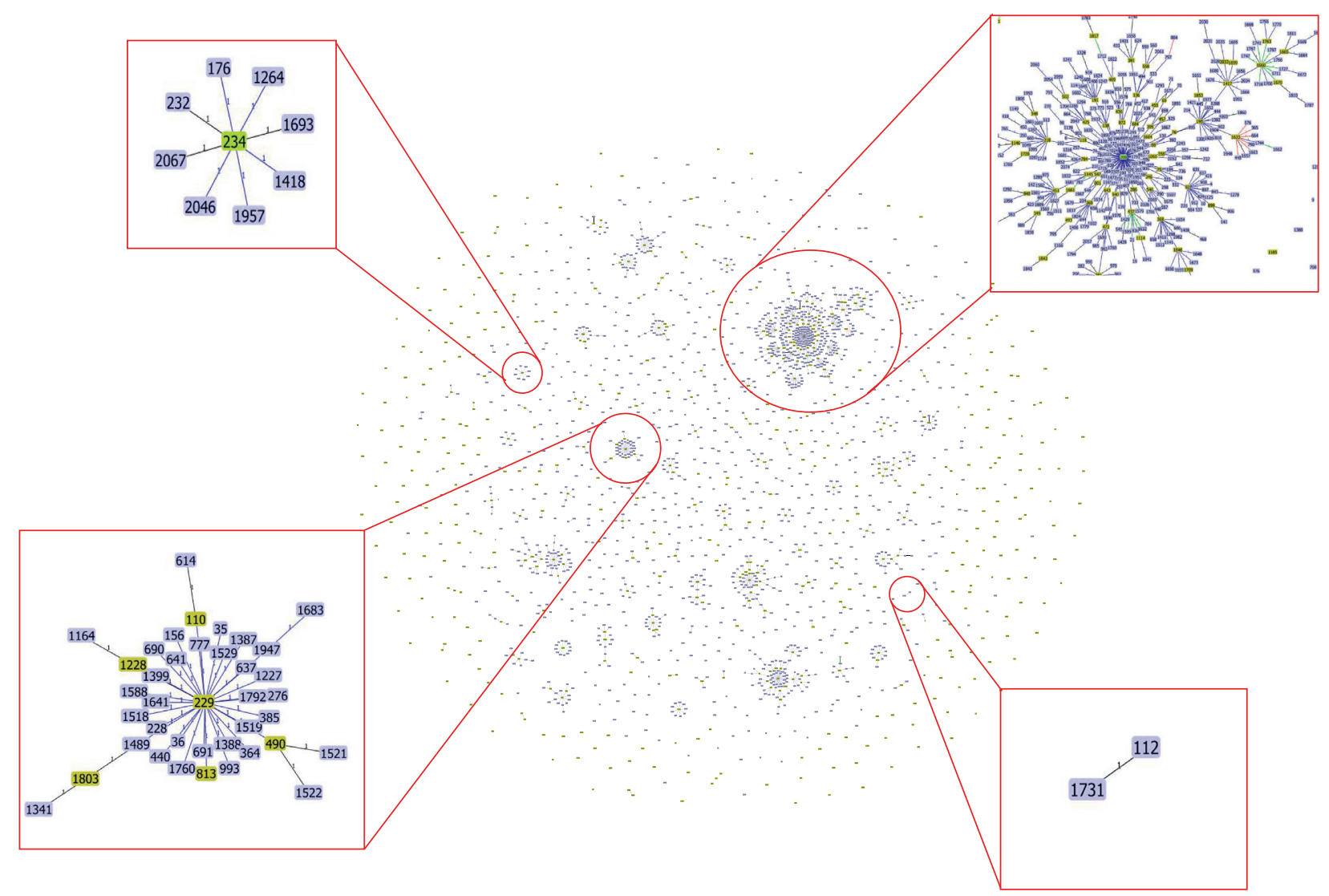

FIgURE 1: Population snapshot of A. baumannii. Clusters of related STs and individual unlinked STs within the entire A. baumannii Oxford MLST database are displayed as a single eBURST diagram by setting the group definition to zero of seven shared alleles. Clusters of linked isolates correspond to clonal complexes. STs found in this current study are magnified in the box beside. A. baumannii strain ATCC 17978 (reference) belongs to ST112.

used to identify pan-genome single-nucleotide polymorphism (SNP) [22]. A SNP phylogenetic tree was drawn based on pairwise whole-genome sequence via Type Genome Server using multiple reference strains that belong to the Acinetobacter baumannii complex group. Ugene-PRO and ISfinder applications were used to analyze the presence of mobile genetic elements [23]. The sequence of this wholegenome shotgun project has been deposited in GenBank under Genome submission: SUB5536145 with the BioProject ID: PRJNA539835.

\section{Results}

The genome sequence size of the 13 isolates in this study ranged from $3,8321,210$ to $4,246,682$ base pair (bp), with contigs ranging from 70 to 104, which encodes 3577 to 4003 coding sequences, 50 to 53 tRNA, 3 rRNA, and 1 tmRNA. Six of $13(46 \%)$ isolates were obtained from endotracheal aspirate followed by urine culture $(4(31 \%))$, rectal swab (2 $(15.4 \%))$, and wound swab (1 (7.7\%)). No isolate of CrAb was cultured from blood samples. Average nucleotide identity (ANI) of all isolates was above 95\% which concludes that they belonged to the same species of bacteria. However, 3 paired isolates shared $100 \%$ identity of ANI although obtained from different sources and states. Those isolates were
CRE400/16-CRE245/15, CRE645/16-CRE648/15, and CRE 1071/16-CRE1159/16. Isolates from our study were compared with a genome of reference strain. The SNP-based phylogenetic tree showed that most of the isolate genomes were closely associated with each other and belonged to international clone II.

MLST analysis with the Oxford scheme in this study revealed a total of 4 defined STs and 2 novel STs (Figure 1). The 2 novel STs of year 2014-2015 were submitted and were assigned as ST1947 and ST1948. ST195, accounting for the largest proportion $(5 / 13,38 \%)$, was the major clonal type followed by ST208 (3/13, 23\%), ST938 (2/13, 15.4), ST1418 $(1 / 13,7.7 \%)$, ST1947 (1/13, 7.7\%), and ST1948 (1/13, 7.7\%). Additionally, ST195, ST208, ST938, and ST1948 were double-locus variants of $g y r B, g b h B$, and $g p i$ genes interchangeably. eBURST analysis showed that these 3 defined STs along with one novel ST clustered in the same CCs (CC92), which was also referred to as global clonal 2 (GC2)/ international clonal II (ICII).

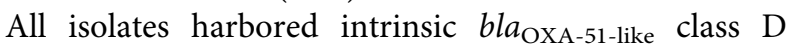
carbapenemases. bla $a_{\text {OXA-66 }}$ was the most prevalent $11(85 \%)$, followed by $b l a_{\mathrm{OXA}-64}$ and $b l a_{\mathrm{OXA}-91}, 1$ (8\%) of each, respectively. An extrinsic bla $a_{\text {OxA-type carbapenemase gene }}$ found was bla $a_{\text {OXA-23 }}(100 \%)$, while no isolates contained bla $a_{\mathrm{OXA}-24-\text { like }}$ or bla $a_{\mathrm{OXA}-58-\text { like }}$ gene. Interestingly, all isolates 


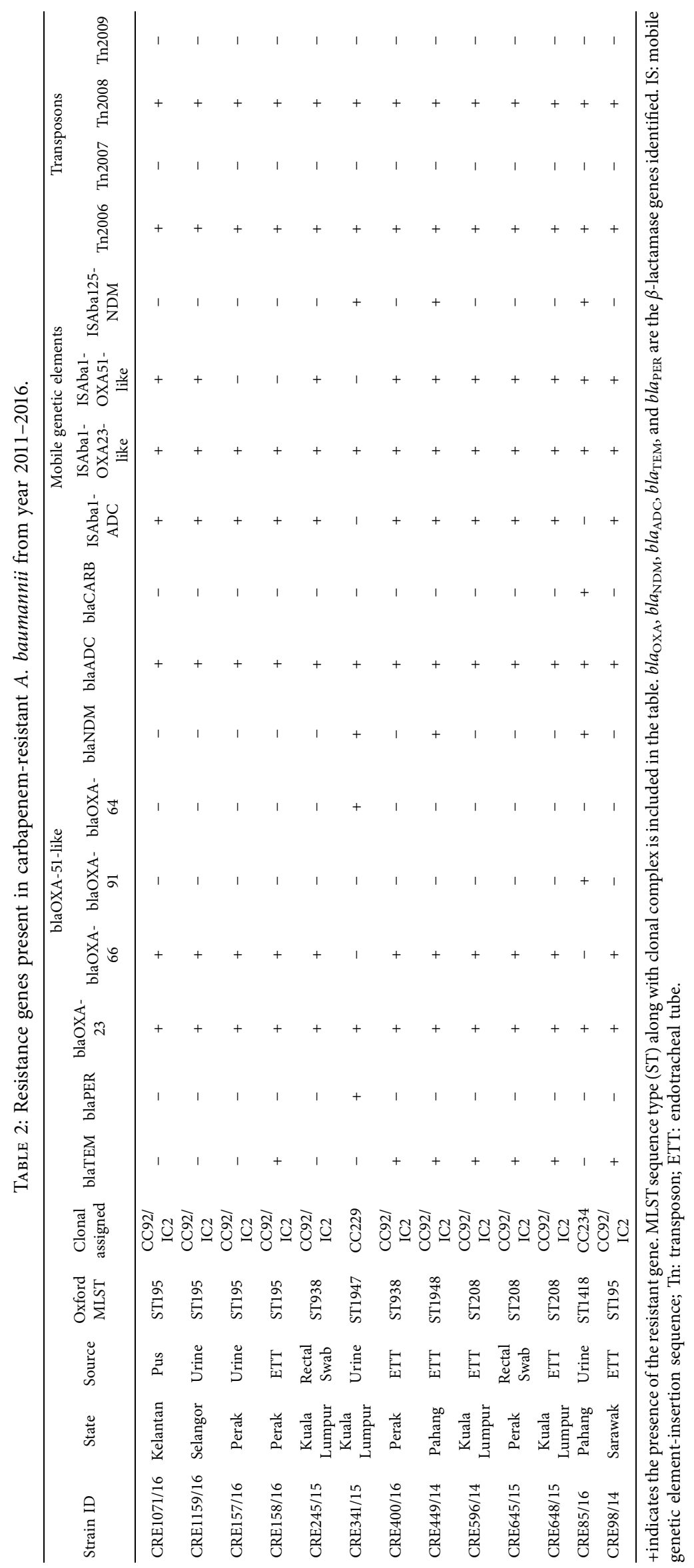




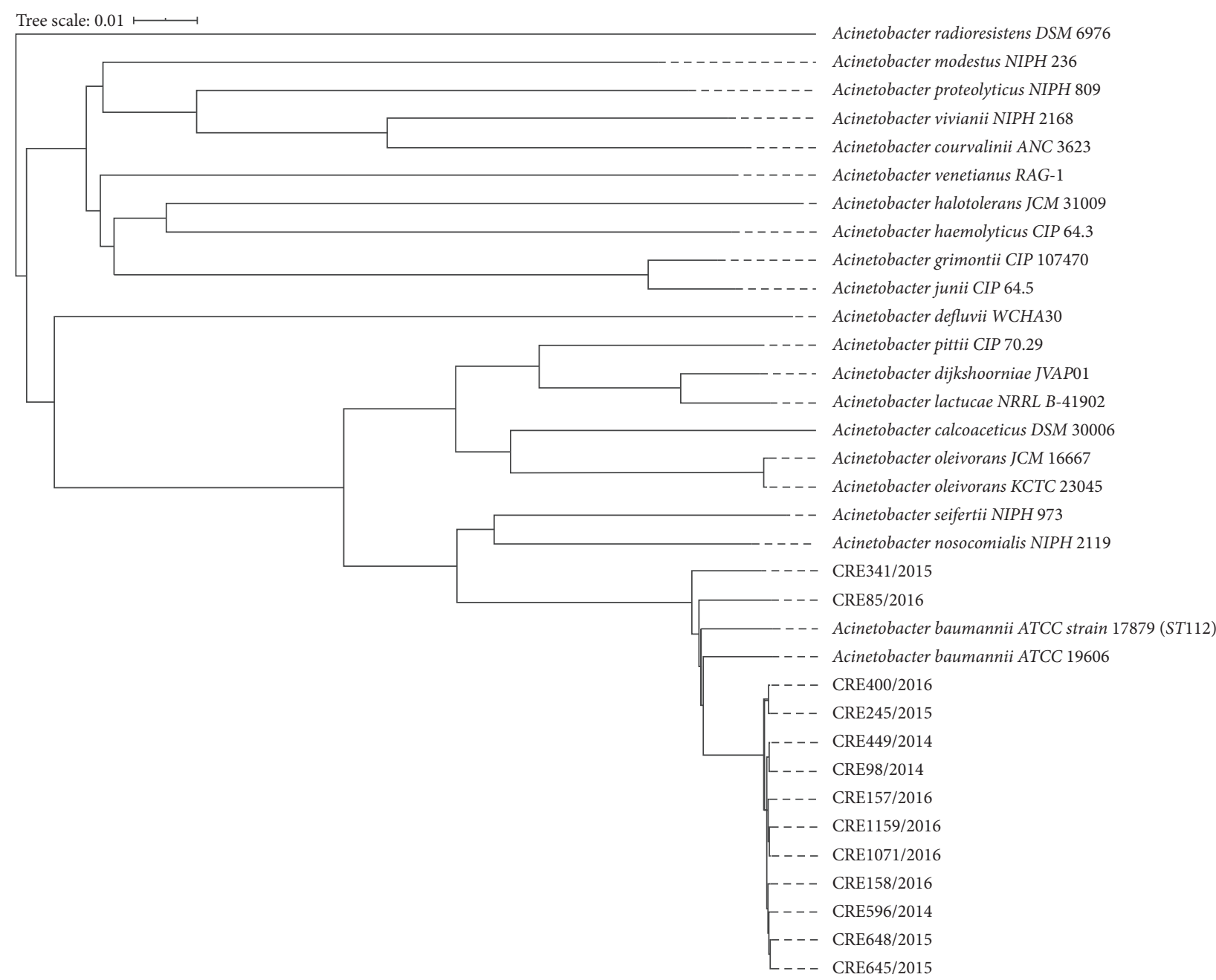

Figure 2: Phylogenetic tree of single nucleotide polymorphisms from whole-genome sequencing was drawn based on pairwise comparison via Type Genome Server. It includes reference strains of other Acinetobacter baumannii complex.

harbored more than one oxacillinase gene. Acinetobacterderived single-variant cephalosporinase $b l a_{\mathrm{ADC}}$ gene was present in all our isolates. A total of $8(62.5 \%)$ isolates harbored class A $\beta$-lactamase gene $b l a_{\mathrm{TEM}}, 1$ isolate carried $b l a_{\mathrm{CARB}}$, and 1 isolate carried bla $a_{\mathrm{PER}}$ gene. In addition, 3 (23.1\%) isolates carried class B metallo- $\beta$-lactamase (MBL) gene $b l a_{\mathrm{NDM}}$. Additionally, all isolates were negative for other MBL genes which included $b l a_{\mathrm{IMP}}, b l a_{\mathrm{VIM}}, b l a_{\mathrm{GIM}}$, and $b l a_{\text {SPM }}$. Table 2 summarizes all the AMR genes detected in this study.

As described in [24], we found that 1 (7.7\%) isolate harbors class 1 integron. The presence of mobile genetic element provides strong evidence for the horizontal dissemination of antibiotic resistance genes. At the same time,

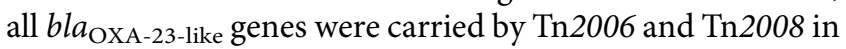
our study. In addition, we detect insertion sequence (IS) elements in the promoter regions of several AMR genes [25]. All isolates with $b l a_{\mathrm{NDM}}$ gene were found to harbor ISAba125 upstream to the corresponding gene $b l a_{\mathrm{NDM}}$. ISAba1 was found upstream to $b l a_{\text {OXA-23 }}(n=13), b l a_{\mathrm{ADC}}$ $(n=11), b l a_{\mathrm{OXA}-66}(n=1)$, and $b l a_{\mathrm{CARB}}(n=1)$. IS91 was found upstream to $b l a_{\mathrm{PER}}(n=1)$.

\section{Discussion}

A. baumannii of nosocomial origin has been the leading cause of hospital-acquired infections [1]. The nature of this bacterium is that it can be found in the environment, intrinsically carrying the antibiotic resistance gene and posing a significant threat to public health due to its unpredictable antibiotic susceptibility $[2,4,5]$. This study was aimed to determine the genetic mechanisms conferring carbapenem resistance in our local strains.

Most of the clinical isolates in this study obtained from respiratory secretion (tracheal aspirate, sputum, and bronchial alveolar lavage) were similar to the Malaysian local surveillance study. Up to year 2017, nosocomial A. baumannii was commonly isolated from respiratory secretion, followed by blood isolation [11,26]. Likewise, many studies nationwide shared similar findings. A. baumannii preferably colonizes or infects the respiratory tract. Such infection commonly occurs in debilitated patients especially in the ICU. Patients of mechanical ventilation and lengthy hospital stay are at risk of $A$. baumannii infection $[27,28]$. 

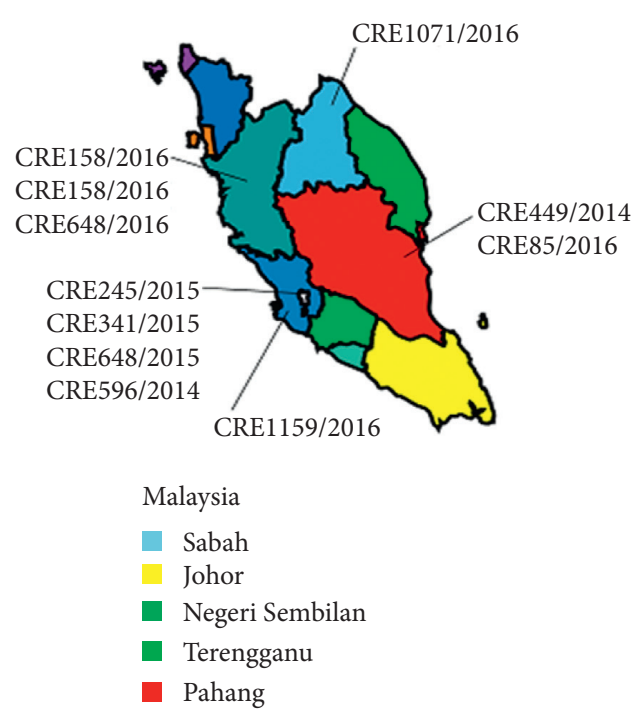
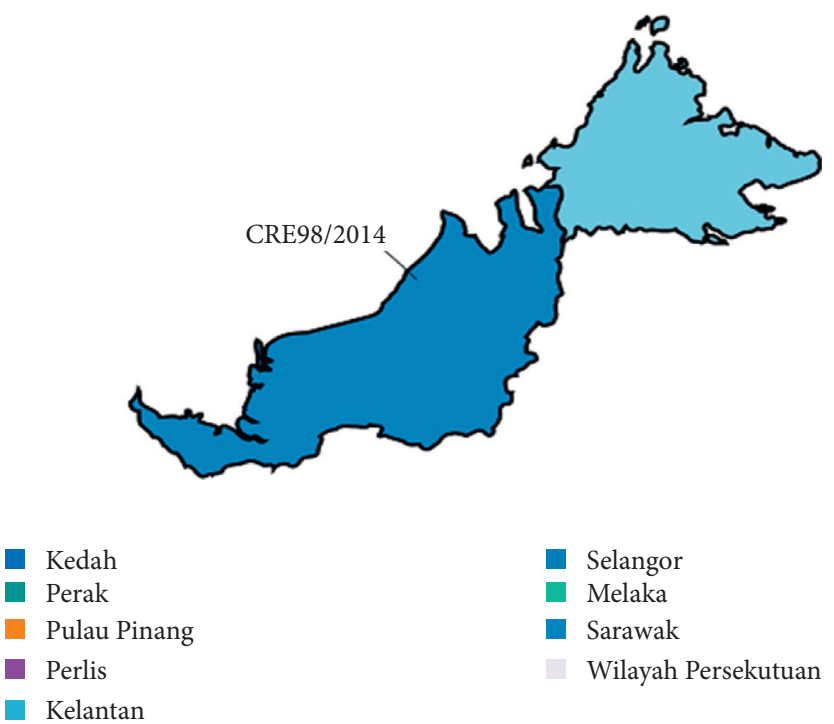

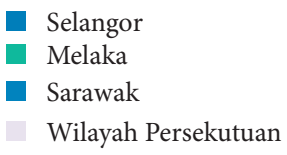

Kelantan

FIGURE 3: Geographic distribution of carbapenem-resistant A. baumannii strains according to different states in Malaysia from year 2011-2016.

ST195 was the frequent sequence types observed in our study. At the same time, as expected, most of these $\mathrm{CrAb}$ isolates belong to the CC92/IC2 clonal lineage. The predominance of CC $92 / \mathrm{IC} 2$ in the present study was similar to reports produced in other neighbouring Asian countries such as China and Thailand and consistent with local studies and reports [29-31]. We also found novel strains of different clonal lineage emerging. Based on our study, these new strains have emerged in 2014 and 2015. In [19, 28, 32], the authors have revealed that emergence of newer strains is caused by inappropriate antibiotic usage. It is crucial to study about epidemiology of sequence types as there is positive correlation between clonal complex and blaOXA carriage. Single-locus sequence-based typing of bla $a_{\text {OXA-51-like }}$ genes assigns 11 clinical samples of this study to a single clonal complex [33]. CC92 clonal lineage isolates commonly harbor $b l a_{\text {OXA-23 }}$ and $b l a_{\text {OXA-66, }}$, similar to the findings in this study [34]. Figures 1-3 portray the SNP phylogenetic tree, minimum spanning tree (MST), and the distribution of the studied isolates in Malaysia.

In clinical microbiology laboratories, A. baumannii is indistinguishable with other species of the Acinetobacter calcoaceticus-Acinetobacter baumannii complex by widely used routine identification systems due to its similar phenotypic and biochemical properties. The accurate identification of A. baumannii is only possible via molecular methods. Molecular characterization of $b l a_{\text {OXA-51-like }}$ gene detection is carried out along with RNA polymerase $\beta$-subunit gene $(r p o B)$ and DNA gyrase B gene $(g y r B)$ for $A$. baumannii species identification [35]. All the isolates involved in this study were positive for $b l a_{\mathrm{OXA}-51-\text { like }}, r p o \mathrm{~B}$, and gyrB genes. This finding resonates along with SNP phylogeny and ANI on species-level identification in this study.

A. baumannii is known for its enzymatic degradation mechanism by $\beta$-lactamases [36]. The most common carbapenem resistance mechanism found in our study was the existence of various $\beta$-lactamases and mobile genetic elements. $b l a_{\text {OXA-23-like }}$ and $b l a_{\text {OXA-51-like }}$ were the most prevalent, accounting for $100 \%$ carbapenem resistance amongst studied isolates. $b l a_{\text {OXA-51-like }}$ gene was detected in all the isolates due to its chromosomal-borne nature, naturally occurring in oxacillinase gene [37].

Meanwhile, $b l a_{\text {OXA-23-like }}$ gene can be either plasmid or chromosome-borne, resulting in increased rates of carbapenem resistance in healthcare settings due to its mobility in facilitating horizontal genetic transfer. The acquisition of $b l a_{\text {OXA-23-like }}$ gene is a major public health concern for its horizontal dissemination and rapid spread [38].

No isolates harbored $b l a_{\text {OXA-24-like, }} b l a_{\text {OXA-48-like, }}$ and $b l a_{\text {OXA-58-like }}$ genes. Although these genes are disseminated in Europe and Middle East, they remained rare in our local findings $[39,40]$. A variant of $b l a_{\text {OXA-51-like }}$ found in this study, namely, bla $a_{\text {OXA-66 }}$ is commonly found in China [41]. At the same time, the present study observed that both $b l a_{\text {OXA-23-like }}$ and $b l a_{\text {OXA-51-like }}$ genes are found in all the isolates. These findings were similar to [42] as it common to find $A$. baumannii isolates harbor $b l a_{\text {OXA-23-like }}$ and $b l a_{\text {OXA- }}$ 51-like in the Asian continent, whereas $b a_{\text {OXA-51-like }}$ and $b l a_{\text {OXA-58-like }}$ in the western hemisphere.

MBL carrying A. baumannii isolates are rare nationwide. $b l a_{\mathrm{NDM}}$ carrying A. baumannii is not commonly found in our area of study. We have not encountered $b l a_{\mathrm{NDM}}$ during previous years or among many local studies [38, 39]. However, to our surprise, a small number $(3 / 13,24 \%)$ of $A$. baumannii isolates collected during 2014-2016 were positive for $b l a_{\mathrm{NDM}}$, indicating recent emergence. Worthy of mentioning is the fact that the isolates carrying $b l a_{\mathrm{NDM}}$ gene did not belong to the same clone lineage [43]. This will be the first reporting on $b l a_{\mathrm{NDM}}$ gene harboring $A$. baumannii isolates from clinical samples in Malaysia.

Similar to the $b l a_{\mathrm{OXA}-51-\text { like }}$ oxacillinase gene, $b l a_{\mathrm{ADC}}$ is also a chromosomally encoded acinetobacter-derived 
cephalosporinase gene found among all the A. baumannii isolates in this study. This finding indicates intrinsic-species specific gene [44]. bla $a_{\text {TEM }}$ genes were also found in $3 / 4$ of our isolates. In [45], the authors had demonstrated that carbepenem resistance among $\mathrm{CrAb}$ is due to the coexistence of $b l a_{\text {OXA-23 }}$ and $b l a_{\text {TEM. }}$. Similar output was observed in this study; however, isolates without $b a_{\text {TEM }}$ also exhibit the resistant phenotypes. To a moderate level, bla $a_{\mathrm{CARB}}$ and $b l a_{\text {PER }}$ were also detected. No prevalence of colistin-resistant genes was found among these isolates. However, there are $A$. baumannii isolates found in many case reports from neighbouring countries such as Taiwan, India, and China that are resistant and becoming resistant [46].

CRE341/15 isolate harbors integrase class 1 indicating sporadic clones. Isolates carrying mobile elements such as integron-encoded integrase gene flanking resistance genes are capable of acquiring and transferring virulence genes via recombination [47]. Transposon played a major role in dissemination of resistant genes. In this study, we observed the presence of transposon $\operatorname{Tn} 2006 / 2008$ in all the isolates carrying $b l a_{\text {OXA-23 }}$ gene. This finding suggests that bla $a_{\text {OXA-23 }}$ dissemination might be due to transposition of transposon [48]. In [14], the authors demonstrated that insertion of IS elements upstream to the resistant genes changes the expression level leading to the increased antimicrobial resistance phenotype. Every isolates in this study was found to have ISAba1 upstream to bla $a_{\mathrm{OXA}-23}$, $b l a_{\mathrm{OXA}-66}, b l a_{\mathrm{ADC}-7}$, and $b l a_{\mathrm{CARB}}$. The ISAba125 was also found in the promoter region of all $b l a_{\mathrm{NDM}}$ positive isolates. These findings suggest that the isolates may have other additional mechanisms resistance against carbapenem [49-51].

In summary, we demonstrated the possible clones of A. baumannii resistant to carbapenem and the prevalence of antibiotic resistance genes associated with mobile genetic elements. These findings provide epidemiological data of prevalent local STs as they are getting more diverse and resistant to multiple antibiotics. The presence of insertion sequence may reflect that these organisms readily take up external DNA. These findings are worrisome for its capability of outbreaks and horizontal resistance gene transmission. Molecular surveillance and antimicrobial stewardship are essential in implementing policies to prevent and control the spread of $\mathrm{CrAb}$ in hospital settings.

\section{Data Availability}

Genome database of this study is available in National Center of Biotechnology Information, NCBI.

\section{Additional Points}

Highlights. (i) The spread of carbapenem-resistant Acinetobacter baumannii is gaining global attention. (ii) It is an opportunistic pathogen that poses a significant threat to public health and is associated with high mortality. (iii)
Selection of an appropriate empirical antimicrobial agent is extremely difficult due to its unpredictable susceptibility patterns. (iv) The association of a mobile genetic element with the resistant gene is worrisome and presents as an emerging threat to our healthcare settings.

\section{Ethical Approval}

This study obtained approval from the National Health Institute Human Research Ethics Committee.

\section{Conflicts of Interest}

All authors declare that they have no conflicts of interest.

\section{Acknowledgments}

This study was supported by a grant from Ministry of Health, Malaysia (NMRR 18-203-40147). The authors are grateful to the Institute for Medical Research, Kuala Lumpur, and the hospitals in Malaysia for their support and facilities. The authors would like to thank the Director General of Health Malaysia for allowing us to publish our findings.

\section{References}

[1] P. E. Fournier, H. Richet, and R. A. Weinstein, "The epidemiology and control of acinetobacter baumannii in health care facilities," Clinical Infectious Diseases, vol. 42, no. 5, pp. 692-699, 2006.

[2] H. J. Doughari, P. A. Ndakidemi, I. S. Human, and S. Benade, "The ecology, biology and pathogenesis of Acinetobacter spp.: an overview," Microbes and Environments, vol. 26, no. 2, pp. 101-112, 2011.

[3] Z. A. Kanafani and S. S. Kanj, Acinetobacter Infection: Treatment and Prevention, UpToDate, Waltham, MA, USA, 2019, https://www.uptodate.com/contents/acinetobacterinfection-treatment-and-prevention.

[4] A. Abbo, S. Navon-Venezia, O. Hammer-Muntz, T. Krichali, Y. Siegman-Igra, and Y. Carmeli, "Multidrug-ResistantAcinetobacter baumannii," Emerging Infectious Diseases, vol. 11, no. 1, pp. 22-29, 2005.

[5] L. A. Gallagher, E. Ramage, E. J. Weiss et al., "Resources for genetic and genomic analysis of emerging pathogen Acinetobacter baumannii," Journal of Bacteriology, vol. 197, no. 12, pp. 2027-2035, 2015.

[6] E. Tacconelli, E. Carrara, A. Savoldi et al., "Discovery, research, and development of new antibiotics: the WHO priority list of antibiotic-resistant bacteria and tuberculosis," The Lancet Infectious Diseases, vol. 18, no. 3, pp. 318-327, 2018.

[7] L. L. Maragakis, T. M. Perl, and T. M. Perl, “Antimicrobial resistance:acinetobacter baumannii:epidemiology, antimicrobial resistance, and treatment options," Clinical Infectious Diseases, vol. 46, no. 8, pp. 1254-1263, 2008.

[8] J. Rello, V. Kalwaje Eshwara, L. Lagunes et al., "A global priority list of the TOp TEn resistant Microorganisms (TOTEM) study at intensive care: a prioritization exercise based on multi-criteria decision analysis," European Journal of Clinical Microbiology \& Infectious Diseases, vol. 38, no. 2, pp. 319-323, 2019.

[9] R. Freeman, L. S. P. Moore, A. Charlett, H. Donaldson, and A. H. Holmes, "Exploring the epidemiology of carbapenem- 
resistant Gram-negative bacteria in west London and the utility of routinely collected hospital microbiology data," Journal of Antimicrobial Chemotherapy, vol. 70, no. 4, pp. 1212-1218, 2014.

[10] R. A. Moghnieh, Z. A. Kanafani, H. Z. Tabaja, S. L. Sharara, L. S. Awad, and S. S. Kanj, "Epidemiology of common resistant bacterial pathogens in the countries of the Arab League," The Lancet Infectious Diseases, vol. 18, no. 12, pp. e379-e394, 2018.

[11] Institute for Medical Research, National Antibiotic Resistance Surveillance Report 2017, https://www.imr.gov.my/images/ uploads/NSAR/NSAR_2017/NSAR_report_2017-edited-31.1. 2019.pdf, Institute for Medical Research, Kuala Lumpur, Malaysia, 2017, https://www.imr.gov.my/images/uploads/NSAR/ NSAR_2017/NSAR_report_2017-edited-31.1.2019.pdf.

[12] B. H. Kong, Y. A. Hanifah, M. Y. M. Yusof, and K. L. Thong, "Antimicrobial susceptibility profiling and genomic diversity of multidrug-resistant Acinetobacter baumannii isolates from a teaching hospital in Malaysia," Japanese Journal of Infectious Diseases, vol. 64, no. 4, pp. 337-340, 2011.

[13] H.-Z. Zhang, "The Acinetobacter baumannii group: a systemic review," World Journal of Emergency Medicine, vol. 4, no. 3, p. 169, 2013.

[14] J. F. Turton, M. E. Ward, N. Woodford et al., "The role of ISAbal in expression of OXA carbapenemase genes in Acinetobacter baumannii," FEMS Microbiology Letters, vol. 258, no. 1, pp. 72-77, 2006.

[15] P. G. Higgins, F. J. Pérez-Llarena, E. Zander, A. Fernández, G. Bou, and H. Seifert, "OXA-235, a novel class D $\beta$-lactamase involved in resistance to carbapenems in acinetobacter baumannii," Antimicrobial Agents and Chemotherapy, vol. 57, no. 5, pp. 2121-2126, 2013.

[16] P. D. Mugnier, L. Poirel, T. Naas, and P. Nordmann, "Worldwide dissemination of theblaOXA-23Carbapenemase gene ofAcinetobacter baumannii1," Emerging Infectious Diseases, vol. 16, no. 1, pp. 35-40, 2010.

[17] A. Bankevich, S. Nurk, D. Antipov et al., "SPAdes: a new genome assembly algorithm and its applications to single-cell sequencing," Journal of Computational Biology, vol. 19, no. 5, pp. 455-477, 2012.

[18] L. M. Rodriguez-R and K. T. Konstantinidis, "Bypassing cultivation to identify bacterial species," Microbe Magazine, vol. 9, no. 3, pp. 111-118, 2014.

[19] S. G. Bartual, H. Seifert, C. Hippler, M. A. D. Luzon, H. Wisplinghoff, and F. Rodriguez-Valera, "Development of a multilocus sequence typing scheme for characterization of clinical isolates of Acinetobacter baumannii," Journal of Clinical Microbiology, vol. 43, no. 9, pp. 4382-4390, 2005.

[20] B. G. Hall, "Building phylogenetic trees from molecular data with MEGA," Molecular Biology and Evolution, vol. 30, no. 5, pp. 1229-1235, 2013.

[21] B. Jia, A. R. Raphenya, B. Alcock et al., "CARD 2017: expansion and model-centric curation of the comprehensive antibiotic resistance database," Nucleic Acids Research, vol. 45, no. D1, pp. D566-D573, 2017.

[22] S. N. Gardner, T. Slezak, and B. G. Hall, "kSNP3.0: SNP detection and phylogenetic analysis of genomes without genome alignment or reference genome: table 1," Bioinformatics, vol. 31, no. 17, pp. 2877-2878, 2015.

[23] K. Okonechnikov, O. Golosova, and M. Fursov, "Unipro UGENE: a unified bioinformatics toolkit," Bioinformatics, vol. 28, no. 8, pp. 1166-1167, 2012.

[24] J. G. M. Koeleman, J. Stoof, M. W. Van Der Bijl, C. M. J. E. Vandenbroucke-Grauls, and P. H. M. Savelkoul,
"Identification of epidemic strains of Acinetobacter baumannii by integrase gene PCR," Journal of Clinical Microbiology, vol. 39, no. 1, pp. 8-13, 2001.

[25] S. Corvec, N. Caroff, E. Espaze, C. Giraudeau, H. Drugeon, and A. Reynaud, "AmpC cephalosporinase hyperproduction in Acinetobacter baumannii clinical strains," Journal of Antimicrobial Chemotherapy, vol. 52, no. 4, pp. 629-635, 2003.

[26] Ministry of Health Malaysia, National Antibiotic Resistance Surveillance Report 2016, http://www.imr.gov.my/images/ uploads/NSAR/NSAR_2016/NSAR_report_2016.pdf, Ministry of Health Malaysia, Putrajaya, Malaysia, 2016, http://www. imr.gov.my/images/uploads/NSAR/NSAR_2016/NSAR_report_ 2016.pdf.

[27] J. Zhang, L. Chen, J. Wang et al., "Molecular detection of colistin resistance genes (mcr-1, mcr-2 and mcr-3) in nasal/ oropharyngeal and anal/cloacal swabs from pigs and poultry," Scientific Reports, vol. 8, no. 1, pp. 1-9, 2018.

[28] R. Handal, L. Qunibi, I. Sahouri et al., "Characterization of carbapenem-resistant Acinetobacter baumannii strains isolated from hospitalized patients in Palestine," International Journal of Microbiology, vol. 2017, Article ID 8012104, 7 pages, 2017.

[29] S.-S. Lean, C. C. Yeo, Z. Suhaili, and K.-L. Thong, "Wholegenome analysis of an extensively drug-resistant clinical isolate of Acinetobacter baumannii AC12: insights into the mechanisms of resistance of an ST195 clone from Malaysia," International Journal of Antimicrobial Agents, vol. 45, no. 2, pp. 178-182, 2015.

[30] S. Biglari, M. M. Rahman, R. Ramliza, and H. Alfizah, "Molecular characterization of carbapenemase and cephalosporinase genes among clinical isolates of Acinetobacter baumannii in a tertiary medical centre in Malaysia," Journal of Medical Microbiology, vol. 64, no. 1, pp. 53-58, 2015.

[31] J. Wang, Z. Ruan, Y. Feng et al., "Species distribution of clinical acinetobacter isolates revealed by different identification techniques," PLoS One, vol. 9, no. 8, Article ID e104882, 2014.

[32] L. Diancourt, V. Passet, A. Nemec, L. Dijkshoorn, and S. Brisse, "The population structure of Acinetobacter baumannii: expanding multiresistant clones from an ancestral susceptible genetic pool," PLoS One, vol. 5, no. 4, Article ID e10034, 2010.

[33] S. Pournaras, V. Gogou, M. Giannouli et al., "Single-locussequence-based typing of blaOXA-51-like genes for rapid assignment of Acinetobacter baumannii clinical isolates to international clonal lineages," Journal of Clinical Microbiology, vol. 52, no. 5, pp. 1653-1657, 2014.

[34] R. Saranathan, R. Kumari, R. Kalaivani et al., "Detection of ISAba1 in association with a novel allelic variant of the $\beta$-lactamase ADC-82 and class $\mathrm{D} \beta$-lactamase genes mediating carbapenem resistance among the clinical isolates of MDR A. baumannii," Journal of Medical Microbiology, vol. 66, no. 2, pp. 103-111, 2017.

[35] S. Vijayakumar, I. Biswas, and B. Veeraraghavan, "Accurate identification of clinically important Acinetobacter spp.: an update," Future Science OA, vol. 5, no. 6, pp. FSO395-2056, 2019.

[36] S. Brown and S. Amyes, "OXA $\beta$-lactamases in Acinetobacter: the story so far," Journal of Antimicrobial Chemotherapy, vol. 57, no. 1, pp. 1-3, 2006.

[37] A. Y. Peleg, H. Seifert, and D. L. Paterson, “Acinetobacter baumannii: emergence of a successful pathogen," Clinical Microbiology Reviews, vol. 21, no. 3, pp. 538-582, 2008.

[38] G. Igrejas, J. Teo, M. Akova et al., "Acinetobacter spp. infections in Malaysia: a review of antimicrobial resistance 
trends, mechanisms and epidemiology," Frontiers in Microbiology, vol. 8, p. 2479, 2017.

[39] S. J. Kim, Y.-J. Kim, and K. S. Ko, "Genomic analysis of consecutive Acinetobacter baumannii strains from a single patient," Frontiers in Microbiology, vol. 9, 2018.

[40] S.-S. Lean, Z. Suhaili, S. Ismail et al., "Prevalence and genetic characterization of carbapenem- and polymyxin-ResistantAcinetobacter baumanniiIsolated from a tertiary hospital in terengganu, Malaysia," ISRN Microbiology, vol. 2014, Article ID 953417, 9 pages, 2014.

[41] Z. Ruan, Y. Chen, Y. Jiang et al., "Wide distribution of CC92 carbapenem-resistant and OXA-23-producing Acinetobacter baumannii in multiple provinces of China," International Journal of Antimicrobial Agents, vol. 42, no. 4, pp. 322-328, 2013.

[42] S. Bleves, V. Viarre, R. Salacha, G. P. F. Michel, A. Filloux, and R. Voulhoux, "Protein secretion systems in Pseudomonas aeruginosa: a wealth of pathogenic weapons," International Journal of Medical Microbiology, vol. 300, no. 8, pp. 534-543, 2010.

[43] A. M. Queenan and K. Bush, "Carbapenemases: the versatile -lactamases," Clinical Microbiology Reviews, vol. 20, no. 3, pp. 440-458, 2007.

[44] W.-H. Zhao and Z.-Q. Hu, "Acinetobacter: a potential reservoir and dispenser for $\beta$-lactamases," Critical Reviews in Microbiology, vol. 38, no. 1, pp. 30-51, 2012.

[45] L. Han, J. Lei, J. Xu, and S. Han, "Bla OXA-23-like and bla TEM rather than bla OXA-51-like contributed to a high level of carbapenem resistance in Acinetobacter baumannii strains from a teaching hospital in Xi'an, China," Medicine, vol. 96, no. 48, p. e8965, 2017.

[46] S. Suzuki, M. Ohnishi, M. Kawanishi, M. Akiba, and M. Kuroda, "Investigation of a plasmid genome database for colistin-resistance gene mcr-1," The Lancet Infectious Diseases, vol. 16, no. 3, pp. 284-285, 2016.

[47] J. F. Turton, N. Woodford, J. Glover, S. Yarde, M. E. Kaufmann, and T. L. Pitt, "Identification of acinetobacter baumannii by detection of the blaOXA-51-like carbapenemase gene intrinsic to this species," Journal of Clinical Microbiology, vol. 44, no. 8, pp. 2974-2976, 2006.

[48] Y. Chen, J. Gao, H. Zhang, and C. Ying, "Spread of the blaOXA-23-containing Tn2008 in carbapenem-resistant Acinetobacter baumannii isolates Grouped in CC92 from China," Frontiers in Microbiology, vol. 8, pp. 1-6, 2017.

[49] Y. Pfeifer, G. Wilharm, E. Zander et al., "Molecular characterization of blaNDM-1 in an Acinetobacter baumannii strain isolated in Germany in 2007," Journal of Antimicrobial Chemotherapy, vol. 66, no. 9, pp. 1998-2001, 2011.

[50] T. J. B. de Man, J. D. Lutgring, D. R. Lonsway et al., "Genomic analysis of a pan-resistant isolate ofKlebsiella pneumoniae, United States 2016," mBio, vol. 9, no. 2, 2018.

[51] K. M. Raible, B. Sen, N. Law et al., "Molecular characterization of $\beta$-lactamase genes in clinical isolates of carbapenem-resistant Acinetobacter baumannii," Annals of Clinical Microbiology and Antimicrobials, vol. 16, no. 1, p. 75, 2017. 